\title{
TDAH: Transtorno e Déficit de Atenção e Hiperatividade
}

\author{
ADHD: Attention Deficit Disorder and Hyperactivity \\ TDAH: Trastorno por Déficit de Atención e Hiperactividad
}

\section{Resumo}

A presente pesquisa tem como objetivos revisar a literatura acerca do TDAH - Transtorno de Déficit de Atenção e Hiperatividade - Analisar o conhecimento dos profissionais acerca do TDAH; e através de revisão literária analisar os problemas que os alunos enfrentam por serem portadores de TDAH. Durante a pesquisa responder à pergunta problema de qual a importância da assistência de enfermagem voltada aos cuidados de alunos com TDAH? A metodologia será a bibliográfica através de artigos, livros eletrônicos, revistas e monografias já publicados na internet a fim de se alcançar o resultado esperado. Inicia-se com uma breve abordagem sobre o Transtorno de Déficit de Atenção e Hiperatividade através da revisão da literatura acerca do tema, trazendo para a pesquisa posicionamentos relacionados ao TDAH dos especialistas tais como alguns autores como QUINTERO, (2011), Martínez, (2019), LEITE e OLIVEIRA, (2015) e Richter (2012). Os principais resultados encontrados foram: que as formas de tratamento podem ser várias, iniciandose com psicoterapias e fármacos, também se procuram realizar intervenções a respeito da vida social, realizando conversas com pais, sobre como colocar em ação algumas estratégias, para ajudar a lidar com os seus filhos.

Palavras-chave: TDAH; Enfermagem; Diagnóstico; Cuidados com os alunos.

\section{Abstract}

This research aims to review the literature on ADHD - Attention Deficit Hyperactivity Disorder - Analyze the knowledge of professionals about ADHD; and through literary review to analyze the problems that students face for having ADHD. During the research, answer the question of what is the importance of nursing care focused on the care 
of students with ADHD? The methodology will be bibliographical through articles, electronic books, magazines and monographs already published on the internet in order to achieve the expected result. It begins with a brief approach to Attention Deficit Hyperactivity Disorder by reviewing the literature on the subject, bringing to the research positions related to ADHD by specialists such as some authors such as QUINTERO, (2011), Martínez, (2019), LEITE and OLIVEIRA, (2015) and Richter (2012). The main results found were: that the forms of treatment can be different, starting with psychotherapies and drugs, they also seek to carry out interventions regarding social life, carrying out conversations with parents, about how to put into action some strategies to help deal with your children.

Keywords: ADHD; Nursing; Diagnosis; Care for students.

\section{Resumen}

Esta investigación tiene como objetivo revisar la literatura sobre TDAH - Trastorno por déficit de atención con hiperactividad - Analizar el conocimiento de los profesionales sobre el TDAH; ya través de la revisión literaria para analizar los problemas que enfrentan los estudiantes por tener TDAH. Durante la investigación, responder a la pregunta de ¿cuál es la importancia de los cuidados de enfermería enfocados en el cuidado de los estudiantes con TDAH? La metodología será bibliográfica a través de artículos, libros electrónicos, revistas y monografías ya publicados en Internet para lograr el resultado esperado. Se inicia con una breve aproximación al Trastorno por Déficit de Atención con Hiperactividad a través de una revisión de la literatura sobre el tema, trayendo a los puestos de investigación relacionados con el TDAH por parte de especialistas como algunos autores como QUINTERO, (2011), Martínez, (2019), LEITE y OLIVEIRA, (2015) y Richter (2012). Los principales resultados encontrados fueron: que las formas de tratamiento pueden ser diferentes, partiendo de psicoterapias y fármacos, también buscan realizar intervenciones en la vida social, llevando a cabo conversaciones con los padres, sobre cómo poner en acción algunas estrategias para ayudar a afrontar el problema. tus niños.

Palabras clave: TDAH; Enfermería; Diagnóstico; Cuidado de los estudiantes.

\section{Introdução}

O presente artigo é uma pesquisa voltada ao TDAH (Transtorno de Déficit de Atenção e Hiperatividade) onde alunos tem dificuldade em permanecer com a atenção voltada ao que está sendo administrado pelo professor, devido uma oscilação de atenção que o aluno portador da TDAH possui.

A escolha do tema foi devido a sua relevância que este tipo de transtorno está tendo atualmente, devido a globalização da educação no mundo, antes crianças que tinha dificuldades escolares não iam para a escola, até mesmo os pais desistiam e a doença não era diagnosticada. Segundo Topczewski: O hiperativo apresenta as dificuldades escolares, o que muito frustra a família. Após um determinado ponto, o professor se torna impaciente e, até intolerante. Por conta dos sucessivos insucessos, começam a se manifestar os conflitos internos, caracterizados pela ansiedade, a qual, pode levar à depressão.

Na pesquisa abordar-se a conceitos sobre TDAH (Transtorno de Déficit de Atenção e Hiperatividade), o qual de acordo com Sena e Neto (2017) O TDAH possui sintomas a serem observados, como fatores que afetam a atenção e a coordenação do comportamento, como motivação, conflitos, ambiente social, estado de consciência e estado psicofisiológico do indivíduo. A atenção é, portanto, resultado de um processo bastante complexo, o qual envolve a percepção temporal e o planejamento, entre outros fatores. O TDAH pode afetar, de forma diferente em cada indivíduo, os diversos aspectos envolvidos no funcionamento da atenção e nas tarefas em que ela é necessária, como no planejamento, na organização e no controle dos estímulos.

A pesquisa justifica-se por ser de extrema importância para a compreensão do estudo do TDAH (Transtorno de Déficit de Atenção e Hiperatividade), um estudo sobre os principais problemas enfrentado por alunos com TDAH. Além disso, se justifica também por procurar proporcionar um maior conhecimento e envolvimento do profissional enfermeiro com a situação observada e refletir sobre o interesse dos profissionais em organizar o seu trabalho, fomentando ações humanizadas na análise da história do paciente.

E nesse sentido, este estudo é relevante por procurar contribuir, de forma consistente, para o desenvolvimento de um corpo de conhecimento próprio em enfermagem relacionado ao TDAH. Sendo, portanto, o tema de máxima importância, uma vez que poucas pessoas sabem a respeito desse assunto 


\section{Metodologia}

$\mathrm{Na}$ presente pesquisa, usou-se métodos específicos para o desenvolvimento do tema proposto, analisando posicionamento obtidos da literatura através de opinião de autores consolidados e assim foi realizado em primeiro momento uma pesquisa bibliográfica, pois: “A pesquisa bibliográfica é elaborada com base em material já publicado, tradicionalmente” (GIL, 2010, p.29).

Para o desenvolvimento desse estudo, será percorrido a metodologia qualitativa, pois conforme Lakatos (2010, p. 269):

A metodologia qualitativa preocupa-se em analisar e interpretar aspectos mais profundos, descrevendo a complexidade do comportamento humano. Fornece análise mais detalhada sobre as investigações, hábitos, atitudes, tendências de comportamentos.

O universo da pesquisa a princípio será feito um levantamento bibliográfico na intenção de coletar informações e dados que servirão de base para a construção da investigação proposta a partir do assunto abordado. Através do uso de materiais já elaborados: livros, artigos científicos e documentos eletrônicos na busca e alocação de conhecimento sobre o tema.

Segundo Gil (2008, p.29):

A pesquisa bibliográfica é elaborada com base em material já publicado. Tradicionalmente, esta modalidade de pesquisa inclui material impresso, como livros, revistas, jornais, teses, dissertações e anais de eventos científicos. Todavia, em virtude da disseminação de novos formatos de informação, estas pesquisas passaram a incluir outros tipos de fontes, como discos, fitas magnéticas, CDs, bem como o material disponibilizado pela Internet.

\section{Resultados e Discussão}

\subsection{O TDAH}

O Transtorno de Déficit de Atenção e Hiperatividade (TDAH) é um transtorno neurocomportamental e multifatorial caracterizado por padrões persistentes de desatenção, desorganização, impulsividade e hiperatividade. Esses indícios estão presentes em pelo menos dois contextos diferentes (casa e escola, por exemplo) e interferem substancialmente no funcionamento social e no desenvolvimento do indivíduo (APA, 2014).

As crianças que possuem desatenção acabam por desenvolver dificuldades em realizar tarefas escolares, é fácil de perder sua concentração, não costumam terminar suas tarefas em casa destinada a elas, não gostam de participar de atividades propostas que envolvam esforço mental, são desorganizadas perdendo seus pertences com frequência. Se estão na frente da TV costumam não responder quando lhe dirigem a palavra, parecendo estar sempre com a cabeça "no mundo da lua" (Argollo, 2013, p. 198).

A impulsividade assim como a desatenção desenvolve certos comportamentos nas crianças que acabam respondendo às perguntas que as pessoas fazem antes de as perguntas serem completadas. Tendem a ter dificuldades em se relacionar com outras crianças, pois não sabem esperar a sua vez na hora de andar em um brinquedo, não sabem respeitar as regras dos jogos querendo sempre ganhar, interferem nas brincadeiras das outras crianças sem serem chamados causando tumultos (ARGOLLO, 2013)

Estima-se que o TDAH possa afetar cerca de 7\% das crianças em idade escolar, números que tem se mostrado estáveis inclusive quando aplicados critérios diagnósticos mais exigentes. Alguns estudos têm estimado que até 10\% das crianças podem ser afetadas (Quintero, 2011). 
Ainda de acordo com diversos estudos, cerca de 75\% das crianças com TDAH serão adolescentes com TDAH e, destes adolescentes, cerca de $50 \%$ serão adultos que seguem apresentando sintomas de TDAH. Neste sentido, é importante a compreensão do conceito chave de que o TDAH é um transtorno que persiste ao longo da vida dos pacientes (Quintero, 2011).

No caso da pessoa com TDAH que não recebeu qualquer tratamento, considera-se que apenas cerca de 10 - $20 \%$ contemplam o que se chama de remissão funcional, ou seja, ausência de manifestações do transtorno com repercussão na vida do indivíduo; para o restante, a evolução será heterogênea, desde adultos funcionais com presença de alguns sintomas que interferem de maneira mínima, até quadros mais complexos com outros transtornos comórbidos associados, como condutas relacionadas ao uso de substâncias e transtornos de personalidade (Quintero, 2016)

Rocha et al. (2010,) pontuam que para a Pedagogia, o TDA/TDAH está relacionado às dificuldades nas percepções e, consequentemente, na aprendizagem. A Psiquiatria explica como sendo um excesso de atividade motora, impulsividade e ausência de atenção. A abordagem comportamental por sua vez procura diagnosticar o transtorno "(...) a partir de uma avaliação do comportamento no ambiente, e analisando quais as situações em que os sintomas aparecem”

$\mathrm{Na}$ atualidade há um consenso estabelecido pelos principais manuais de prática clínica acerca da abordagem multimodal como o padrão ouro de tratamento para o TDAH, que tem como base o estudo MTA - estudo do tratamento multimodal de crianças com TDAH, com as seguintes orientações: tratamento farmacológico combinado à outras medidas, definidas em função das necessidades particulares de cada caso. (Martínez, 2019).

\subsection{A importância do professor e da enfermagem nos cuidados de alunos com TDAH}

Nos dias atuais a educação escolar ainda segue um modelo de ensino hierárquico, onde na maioria das vezes a aprendizagem é mecânica. (LEITE e OLIVEIRA, 2015). Esse tipo de ensino prejudica muito a aprendizagem de alunos com este transtorno.

Porém, embora exista em algumas escolas a falta de conhecimento desse transtorno por alguns professores, outros já notam e comunicam aos pais.

A Política Nacional de Educação Especial na Perspectiva da Educação Inclusiva, datada de 2007, não incluiu os alunos com TDAH dentro da Educação Especial. A partir dessa decisão, surgiram iniciativas da sociedade civil e de organizações pretendendo dar visibilidade a esta parcela do corpo discente. Um exemplo desses grupos é a Associação Brasileira do Déficit de Atenção (ABDA), que tem por objetivo conquistar garantias legais para as pessoas com TDAH. Dentre as metas alcançadas, tem-se o reconhecimento das necessidades especiais destes estudantes no ENEM, fato que promove adaptações no estilo de prova e algumas leis regionais (ABDA, 2017)

Vários processos se encontram em tramitação, incluindo o Projeto de Lei 7081/2010, que precisa ser votado e sancionado pela Presidência da República. Em concomitância, o Ministério Público, através dos parâmetros no ECA (Estatuto do Menor e Adolescente) e do Decreto Legislativo nº 186, de 2008, exposto no Artigo 5º Parágrafo 3, da Constituição Federal, vem proporcionando sentenças favoráveis para as pessoas com TDAH que buscam os seus direitos, inclusive acesso gratuito à medicação, conforme determinado no artigo $11^{\circ}$ (ABDA, 2017).

Com o aumento de crianças com TDAH no ambiente escolar é de grande importância e necessária a discussão em como deve ser trabalhado o ensino para esses, visto que necessitam de uma atenção diferenciada por conta de suas particularidades em relação à assimilação dos conteúdos. É muito comum no sistema de educação brasileiro se deparar com professores em sala de aula, que diagnosticam estudantes que apresentam comportamentos que lembram o TDAH e a partir daí apresentar insegurança por não possuir estratégias pedagógicas que favoreçam a aprendizagem desses estudantes. (Santos \& Vasconcelos, 2010).

Em alguns casos, de crianças que possuem alguma dificuldade de aprendizagem, faz-se, necessário o acompanhamento com uma psicopedagoga. Já em casos mais extremos em que as crianças apresentam morbidades relacionadas aos seus problemas 
como, por exemplo, a depressão, ansiedade e comportamentos disruptivos, é importante que haja um acompanhamento terapêutico tanto para a família como para a criança. Quando a terapia está associada a um ambiente bem estruturado pode-se permitir doses menores de medicamentos (Argollo, 2013).

Segundo (Richter, 2012, p. 38): [...] o medicamento ameniza os sintomas durante cerca de até quatro horas após ser ingerido, podendo apresentar reações adversas como perda de apetite, cefaleia, náuseas, insônia, taquicardia, tonturas, vômitos, psicose e, em alguns casos, perda de peso e diminuição do crescimento, possivelmente em função da perda de apetite ocasionada pelo medicamento.

Foram usados para a pesquisa 4 artigos, o primeiro por título "manejando da melhor maneira possível o tdah" de Dr Javier Quintero, chefe de Servicio de Psiquiatría. Hospital Universitario Infanta Leonor. Universidad Complutense. Madrid. España.

Existem muitos estudos que procuram comprovar que esse transtorno de fato existe e que vem sendo descrito há muito tempo, desde o século XIX, já existem descrições sobre ele. Há também estudos epidemiológicos mostrando que existem taxas semelhantes de TDAH em vários países do mundo, independentemente da cultura em que estão inseridos os sujeitos, as taxas parecem semelhantes, conforme afirma a psiquiatra Rosário em entrevista concedida a Veja (2013, texto digital).

Segundo diversos estudos, cerca de 75\% das crianças com TDAH serão adolescentes com TDAH e, destes adolescentes, cerca de 50\% serão adultos que seguem apresentando sintomas de TDAH. Neste sentido, é importante a compreensão do conceito chave de que o TDAH é um transtorno que persiste ao longo da vida dos pacientes (Quintero, 2016).

O segundo artigo tratando sobre "O transtorno de déficit de atenção e hiperatividade (TDAH) e os desafios no contexto escolar: o lúdico como principal contribuinte de autoria" de Ketlyn Selmer. A pesquisa aponta que "o transtorno junto com a dislexia simboliza o principal motivo de fracasso escolar e está presente em 7\% das crianças no Brasil, sendo tratado como um grave problema de saúde pública. Apesar de o termo TDAH ser pouco conhecido pelos educadores, apresenta um grande número em relação ao fraco desempenho escolar. Trata-se de uma doença neuropsiquiátrica caracterizada por desatenção, hiperatividade e impulsividade, que surge na infância e se estende até a fase adulta. (Couto et al, 2010).

Preocupados com as poucas informações a respeito do tema, no ano de 2002, criou-se uma Declaração de Consenso Internacional sobre o TDAH, iniciada por Russell A. Barkley, professor de Psiquiatria e Neurologia da Universidade da Massachussetts Medical School, EUA, esta foi assinada por mais de 80 profissionais cientistas de diversos países, especializados nesse transtorno. (Partel, 2006)

De acordo com os estudos realizados por Pinheiro, 2010, os sintomas dos tipos listados devem ocorrer frequentemente e servem para tornar o diagnóstico mais padronizado.

Nas pesquisas relacionadas ao TDAH, foram apontadas que os fatores genéticos representam $80 \%$ dos casos, caso os pais possuam o transtorno, há chance de seus filhos desenvolverem, o que acontece também em casos de gerações com transtorno de ansiedade, de humor e alcoolismo. Mesmo o TDAH tendo maior incidência em crianças, o predomínio em adultos é de aproximadamente 4\%, apresentando falta de atenção, intolerância, impulsividade, irritabilidade e frustações. (Poeta \& Rosa Neto, 2014)

Essa pesquisa foi usada muitos outros métodos como vários livros, revistas e monografias, onde pode ser vista nas referências bibliográficas deste artigo.

\section{Conclusão}

Na conclusão deste trabalho, durante a pesquisa procuramos analisar os estudos sobre o transtorno TDAH, o qual temse notícias que começaram nas décadas de 80 e 90, primeiramente nos Estados Unidos, ocorrendo nessa época uma explosão publicitária em torno do diagnóstico do transtorno. 
Onde foi exposto sobre o TDAH, definido por dificuldade de prestar atenção a detalhes ou errar por descuido em atividades escolares, falta de atenção em tarefas ou atividades lúdicas; parecer não escutar quando lhe dirigem a palavra; não seguir instruções e não terminar tarefas escolares, domésticas ou deveres profissionais; dificuldade em organizar tarefas e atividades; enfim dificuldade em envolver-se em tarefas que exijam esforço mental constante e ser facilmente distraído à tarefa e apresentar esquecimentos em atividades diárias.

Dentre os trabalhos encontrados na literatura brasileira, destacam que são milhares de crianças e adultos em todo o Brasil que sofrem desse transtorno e não é difícil perceber esses sintomas nas crianças com o transtorno, elas estão sempre em constantes movimentos, quando sentadas ficam se balançando ou mexendo os pés e mãos, tocam tudo com as mãos, falam demais, sentem menos necessidades de descansar ou dormir, mordiscam, chupam, mordem tudo, como lápis, mangas de camisas, entre outros

No transcorrer da pesquisa analisamos o lado positivo de cada procedimento dos métodos usados pelos professores e da enfermagem nos cuidados de alunos com TDAH. Constatou-se que na maioria das vezes as dificuldades de atenção e hiperatividade são notadas quando a criança ingressa na escola, onde os comportamentos são percebidos pelos professores, quando comparado com outros estudantes da mesma idade e classe.

Assim, o professor é de fundamental importância para o diagnóstico da doença. Dessa forma, o ambiente escolar é de grande importância e necessária a discussão em como deve ser trabalhado o ensino para crianças diagnosticada com TDAH. Às habilidades e competências dos enfermeiros. E que atualmente se inverteu o procedimento e que outros métodos vão sendo implantados para melhorar o tratamento desse transtorno. E que estas estratégias usadas pelos profissionais são de grande valia para conduzir da melhor maneira possível à recuperação dos pacientes.

Depois da pesquisa que envolveu estudo de vários livros e artigos sobre o tema, conclui-se é de fundamental Importância para pais e professores o esclarecimento do transtorno e de como devem lidar com essas crianças diagnosticada com TDAH.

\section{Referências}

ABDA: Associação Brasileira do Déficit de Atenção (2017). Leis municipais e estaduais sobre TDAH. https://tdah.org.br/leis-municipais-eestaduais-sobre-tdah/ Argollo, N. (2013) Transtorno do déficit de atenção com hiperatividade: aspectos neuropsicológicos. Psicologia Escolar e Educacional, 7(2), $197-201$.

Couto, T. S., Melo Junior, M. R., \& Gomes, C. R. A. (2010). Aspectos neurobiológicos do transtorno do déficit de atenção e hiperatividade (TDAH): uma revisão. Ciências \& Cognição, 15(1), 241-251.

Richter, B. R. (2012) Hiperatividade ou indisciplina? - O TDAH e a patologização do comportamento desviante na escola. Dissertação (Mestrado) - UFRGS.

Leite, L. B. M.; \& Oliveira, C. B. E. Atividades Lúdicas no Ensino de Biologia para Alunos que Cumprem Medida Socioeducativa de Internação. X Encontro Nacional de Pesquisa em Educação em Ciências - X ENPEC, 2015.

Martínez-Núñez, B., \& Quintero, J. Update the Multimodal Treatment of ADHD (MTA): twenty years of lessons. Actas Esp Psiquiatr. 2019

Partel, C.H. Universo TDAH. Declaração Internacional de Consenso sobre o TDAH. 2006.

Poeta, L.S. \& Neto, F.R. (2014). Estudo epidemiológico dos sintomas do transtorno do déficit de atenção/hiperatividade e transtornos de comportamento em escolas da rede pública de Florianópolis usando a EDAH. Rev. Bras. Psiquiatr, 2006; Vol.26 n.3, 150.155. < http://www.scielo.br/pdf/rbp/v26n3/a04v26n3.pdf>.

Quintero, J., Loro, M., Jiménez, B., \& García, C. N. (2011) Evolutionary issues in attention deficit hyperactivity disorder (ADHD); from risk factors to comorbidity and social and academic impact. Vertex.

Quintero, J., Martin, M., Alcindor, P., \& Perez-Templado, J. (2016) Prevention in attention deficit hyperactivity disorder]. Rev Neurol.

Santos, L. F.; \& Vasconcelos, L. A. (2010). Transtorno do Déficit de Atenção e Hiperatividade em Crianças: Uma Revisão Interdisciplinar. Psicologia: Teoria e Pesquisa. 26(4), 717-724. < http://www.scielo.br/pdf/ptp/v26n4/15.pdf>. 